\title{
Significance of androgen receptor and CD10 expression in cutaneous basal cell carcinoma and trichoepithelioma
}

\author{
HESNA M. ASTARCI ${ }^{1}$, GULFEM A. GURBUZ ${ }^{2}$, DEMET SENGUL ${ }^{3}$, SEMA HUCUMENOGLU ${ }^{4}$, \\ UGUR KOCER $^{5}$ and HUSEYIN USTUN ${ }^{6}$ \\ ${ }^{1}$ Department of Pathology, Abant Izzet Baysal University, Bolu 14280; ${ }^{2}$ Department of Dermatology, \\ Dr. N.K. Sincan State Hospital, Ankara 06930; ${ }^{3}$ Department of Pathology, Giresun University, Giresun 28200; \\ Departments of ${ }^{4}$ Pathology and ${ }^{5}$ Plastic and Reconstructive Surgery, Ankara Training and Research Hospital, \\ Ankara 06340; ${ }^{6}$ Department of Pathology, Kafkas University, Kars 36100, Turkey
}

Received January 18, 2015; Accepted August 25, 2015

DOI: $10.3892 / \mathrm{ol} .2015 .3804$

\begin{abstract}
Differential diagnosis of trichoepithelioma (TE) and basal cell carcinoma (BCC) on the basis of clinical symptoms and laboratory investigations may be difficult in certain patients. The aim of the present study was to compare cluster of differentiation 10 (CD10) and androgen receptor (AR) expression patterns in $\mathrm{BCC}$ and $\mathrm{TE}$, to investigate the predictive power of these proteins as markers of the two conditions. A total of 39 cases of BCC and 15 cases of TE were retrieved from the pathology department archives. AR and CD10 immunohistochemistry was performed on all of the specimens; 23 BCC cases displayed focal nuclear AR staining, however, none of the cases demonstrated diffuse nuclear staining and 16 BCC cases were negative for AR staining. Stromal CD10 staining was more common in TE cases than in $\mathrm{BCC}$ cases, and peripheral CD10 staining was more common in BCC cases than in TE cases. AR immunostaining of the BCC samples typically appeared as scattered clusters and individual cells. In addition, AR and CD10 staining exhibited varying staining intensities within each samples. Incisional punch biopsy specimens have the potential to present false-negative results. Therefore, AR and CD10 staining of total excision biopsies provides a more accurate differential diagnosis of BCC and TE for cases with difficulties in the histopathological analysis.
\end{abstract}

\section{Introduction}

Basal cell carcinoma (BCC) is the most prevalent cutaneous tumor, and accounts for $70 \%$ of all malignant skin diseases (1). BCCs display a large degree of morphological variability, and

Correspondence to: Dr Hesna M. Astarci, Department of Pathology, Abant Izzet Baysal University, Golkoy Yerleskesi, Bolu 14280, Turkey

E-mail: muzeyyenastarci@yahoo.com.tr

Key words: basal cell carcinoma, trichoepithelioma, CD10, androgen receptor, immunohistochemistry a number of histopathological subtypes have been defined (1). $\mathrm{BCC}$ is an epithelial tumor, which arises from progenitor cells of the interfollicular epidermis and upper infundibulum (2). Three histological subtypes of BCC exist: Superficial, nodular and infiltrative (1). An additional type of epithelial tumor, trichoepithelioma (TE), originates from the outer root sheath of the hair follicle (3). In contrast to BCC, TE is a benign tumor with clear follicular differentiation. Three major variants of trichoepithelioma have been identified, namely the solitary, multiple and desmoplastic subtypes. The histological features of the solitary and multiple subtypes are identical (3). In certain cases, it may be difficult to clinically or microscopically distinguish TE and BCC. It is important to accurately differentiate the two neoplasms, as they exhibit distinct biological behaviors, for example malignancy. Furthermore, each condition has distinct potential treatment strategies, thus an accurate diagnosis is significant for prognosis.

Human sebaceous glands and hair follicles are skin structures targeted by androgen action. These structures contain steroid enzymes capable of transforming weak androgens into the tissue-active androgens testosterone and dihydrotestosterone, which bind to the androgen receptor (AR) and regulate the transcription of target genes (4). The detection of AR (using antibodies) is a promising tool for use in the differention between BCC and TE (5). In normal skin, AR is expressed in the sebaceous glands, interfollicular epidermal keratinocytes, pilosebaceous duct keratinocytes, dermal fibroblasts and secretory coil cells of the eccrine sweat glands (6-8). AR expression has been suggested to occur in a number of cutaneous neoplasms, including BCC (6). By contrast, AR appears not to be expressed in mature hair follicles, the epidermis or benign hair follicle tumors, for example TE (8).

Cluster of differentiation 10 (CD10) is a $100 \mathrm{kDa}$ transmembrane glycoprotein, which was initially identified as common acute lymphoblastic leukemia antigen (9). CD10 expression is associated with cellular growth rates, and is elevated in malignant tumors and regenerating tissues, although its expression is not lineage-specific (10). Furthermore, CD10 expression has been detected in peritumoral fibroblast-like stromal cells within the invasive areas of certain types of cancer, including prostate, breast, colorectal 
and lung carcinomas (11). In healthy adult skin, CD10 immunopositivity is observed in the inner sheath of the hair follicles, hair matrix and perifollicular fibrous sheath (12). Additionally, CD10 is expressed in a number of types of skin cancer, including dermatofibroma, dermatofibrosarcoma protuberans and melanoma (13).

The aim of the present study was to compare the CD10 and AR expression patterns in BCC and TE cases, and to determine whether they may serve as useful diagnostic markers.

\section{Materials and methods}

Case selection. Tumor samples were obtained by total excisional resection, and incisional biopsy specimens were excluded. The data were collected over 2 years, between 2010 and 2012 . Ethical approval for this study was obtained from the Ankara Training and Research Hospital's institutional review board. A total of $39 \mathrm{BCC}$ and $15 \mathrm{TE}$ cases were retrieved from the pathology department archives at the Ankara Training and Research Hospital (Ankara, Turkey) for analysis of immunohistochemical staining patterns. Hematoxylin and eosin (H\&E)-stained sections were reviewed by two pathologists with experience in dermatopathology (Dr Hesna M. Astarci and Professor Huseyin Ustun), and a diagnosis of BCC or TE was confirmed. The 39 BCC samples were classified into 4 groups: Superficial (6 cases), nodular (30 cases), infiltrative (1 case) and mixed (2 cases) (1). Biopsies were obtained from the face and back.

Immunohistochemistry. Immunohistochemical analysis was performed on all of the specimens (54 cases). The present study compared TE with BCC. Formalin (10\% solution; $\mathrm{pH}$ 7.0-7.6)-fixed, paraffin-embedded tumor tissues were prepared for immunohistochemical evaluation. In each case, a pair of 4- $\mu \mathrm{m}$ sections was placed on poly-L-lysine-coated slides (Sigma-Aldrich, St. Louis, MO, USA). The tissue sections were dried for $12 \mathrm{~h}$ in a $37^{\circ} \mathrm{C}$ oven, deparaffinized with xylene and rehydrated using graded alcohol (Merck Millipore, Darmstadt, Germany). Antigen retrieval was performed by heating the slides under pressure with EDTA (ScyTek Laboratories, Inc., Logan, UT, USA) for $9 \mathrm{~min}$. The sections were placed in the aforementioned solutions for $20 \mathrm{~min}$ at room temperature and then placed in phosphate-buffered saline (PBS) solution (ScyTek Laboratories, Inc.). Endogenous peroxidase activity was inhibited by incubation in $1 \% \mathrm{H}_{2} \mathrm{O}_{2}$ for $15 \mathrm{~min}$. Following PBS washes, the samples were incubated with Ultra V Block (ScyTek Laboratories, Inc.) to inhibit non-specific binding. Each pair of tissue sections was incubated for $2 \mathrm{~h}$ at room temperature with mouse monoclonal primary antibody against human CD10/CALLA (neutral endopeptidase) (clone 56C6; \#MS-728-R7; Thermo Fisher Scientific, Fremont, CA, USA). The sections were then washed four times with PBS and incubated for 20 minutes at room temperature with a biotinylated goat anti-polyvalent secondary antibody (UltraTek HRP Anti-Polyvalent Lab Pack; \#UHP125; ScyTek Laboratories, Inc.). The slides were then washed with PBS and incubated in a diaminobenzidine chromogen-substrate (ScyTek Laboratories, Inc.) for $5 \mathrm{~min}$. The sections were subsequently counterstained with Mayer's hematoxylin solution (Sigma-Aldrich), then dehydrated with alcohol and cleared with xylene. Finally, entellan (Merck

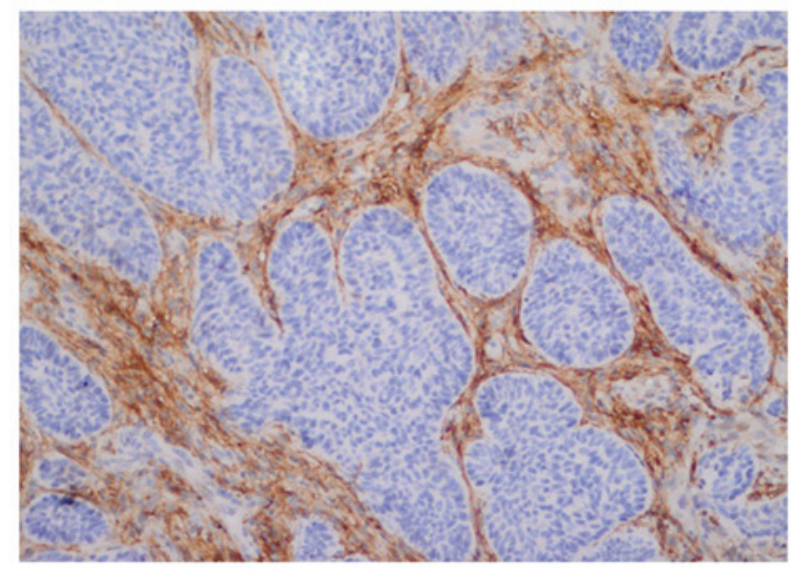

Figure 1. Stromal CD10 immunostaining (brown) surrounding trichoepithelioma tumor cell islands. Tumor cells are negative for CD10 staining (magnification, $\mathrm{x} 40$ ).

Millipore) was added to the slides, and the samples were mounted with coverslips. In each case, human small intestine tissue sections were stained for CD10 for use as a positive control (i6000 Automatic Staining System, Biogenex, Fremont, CA, USA). Anti-CD10 localization to the stroma and/or tumor cells was determined according to the following four immunoreactivity groups: Stromal staining, peripheral staining, central staining and no staining (Fig. 1). Paraffin-embedded blocks were also stained for AR expression using a standard immunoperoxidase technique (using the i6000 Automatic Staining System) (14). In brief, the sections were deparaffinized, and antigen retrieval was performed by heat treatment at $\mathrm{pH} 9.5$ for $3 \mathrm{~min}$ in Borg Decloaker solution (Biocare Medical, Inc., Concord, CA, USA). Sections were incubated overnight at room temperature with a monoclonal mouse anti-human AR antibody (diluted 1:75; clone AR441; \#M3562; Dako North America, Inc., Carpinteria, CA, USA). The sections were subsequently incubated with a goat anti-mouse IgG Biotinylated Universal Link secondary antibody (\#GU600; Biocare Medical, Inc.) for $20 \mathrm{~min}$ and streptavidin-enzyme conjugate (Streptavidin-horseradish peroxidase; Biocare Medical, Inc.) for $20 \mathrm{~min}$ at room temperature. The sections were finally stained with a 3,3'-diaminobenzidine chromogen kit and counterstained with Mayer's hematoxylin. In BCCs, AR expression is frequently distributed focally, therefore, any tumor displaying focal nuclear AR staining was classified as AR-positive (14). The AR-stained specimens required individual interpretation and comparison with an internal positive control (sebaceous glands) on the same slide (Figs. 2 and 3). In BCC, AR expression frequently exhibits a focal and nuclear distribution, and AR-positive immunostaining generally appears as scattered clusters and as individual cells. AR immunoreactivity was classified into the following five groups: Negative, $(<1 \%$ AR positive cells); $1+$, focal positive (1-5\%); $2+$, focal positive $(5-25 \%) ; 3+$, focal positive (25-49\%); and $4+$, diffuse positive ( $>50 \%)$.

Statistical analysis. Data were collected and statistical analysis was performed using SPSS version 20.0 (IBM SPSS, Armonk, NY, USA). Fisher's exact and $\chi^{2}$ tests were used for comparisons between the nominal variables. $\mathrm{P}<0.05$ was considered to indicate a statistically significant difference. 
Table I. CD10 expression patterns in BCC $(n=39)$ and TE $(n=15)$ groups.

\begin{tabular}{lccc}
\hline CD10 staining & BCC, n (\%) & TE, n (\%) & P-value \\
\hline Stromal & $11(28.2)$ & $9(60.0)$ & 0.030 \\
Peripheral & $23(59.0)$ & $4(26.7)$ & 0.033 \\
Central & $10(25.6)$ & $3(20.0)$ & 0.480 \\
None & $7(17.9)$ & $3(20.0)$ & 0.571 \\
\hline
\end{tabular}

Certain tumors expressed more than one pattern of staining. CD10, cluster of differentiation 10; BCC, basal cell carcinoma; TE, trichoepithelioma.

Table II. AR staining in BCC cases $(\mathrm{n}=39)$.

\begin{tabular}{lc}
\hline AR staining, $\%$ & BCC cases, $\mathrm{n}(\%)$ \\
\hline$<1$ & $16(41.0)$ \\
$1-5$ & $12(30.8)$ \\
$6-25$ & $9(23.1)$ \\
$26-50$ & $2(5.1)$ \\
\hline
\end{tabular}

$\mathrm{AR}$, androgen receptor; $\mathrm{BCC}$, basal cell carcinoma.

Table III. Gender ratio of AR (+) basal cell carcinoma cases $(n=23)$.

\begin{tabular}{lc}
\hline Gender & AR $(+)$ cases, $\mathrm{n}(\%)$ \\
\hline Female & $5(21.7)$ \\
Male & $18(78.3)$ \\
\hline
\end{tabular}

AR (+), androgen receptor positive.

\section{Results}

CD10 expression in BCC and TE patients. The present study involved $11(28.2 \%)$ female and $28(71.8 \%)$ male patients with BCC. The age of the patients ranged from 34-89 years, with a mean age of $65.74 \pm 12.388$ years. The patients with TE comprised 7 (46.7\%) females and $8(53.3 \%)$ males. The patients' ages ranged from 24-74 years, with a mean age of $55.40 \pm 21.33$ years. The majority of the BCC cases and all of the TE cases were localized in the head region. The BCC cases were classified into four groups. Furthermore, CD10 expression was classified into four groups and was compared between the BCC and TE groups (Table I). One tumor displayed multiple CD10 staining patterns. A total of 9 TE $(60 \%)$ and 11 BCC cases $(28.2 \%)$ presented stromal CD10 staining. Stromal CD10 staining was significantly more common in TE cases than in BCC cases $(\mathrm{P}=0.030)$. In addition, $23 \mathrm{BCC}(59 \%)$ and $4 \mathrm{TE}$ cases (26.7\%) displayed peripheral CD10 staining. Peripheral CD10 staining was significantly more common in $\mathrm{BCC}$ cases than in TE cases $(\mathrm{P}=0.033)$.

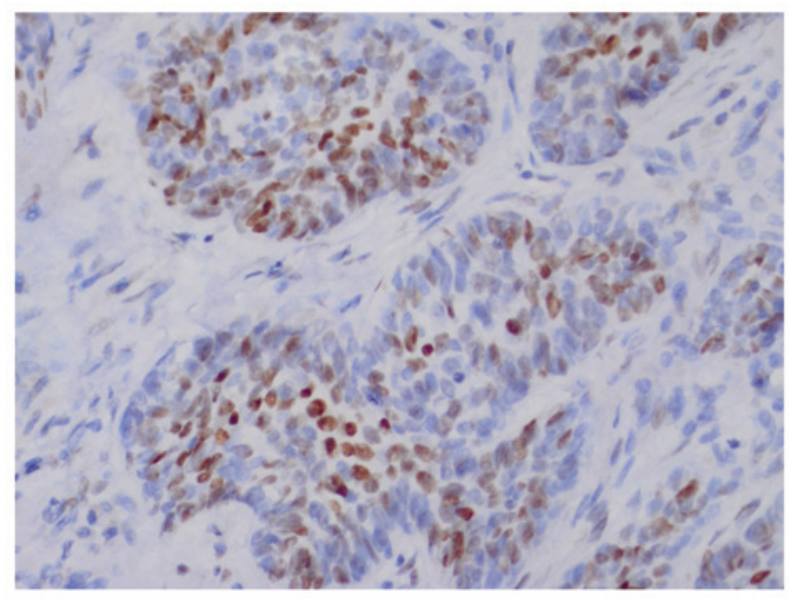

Figure 2. Nuclear androgen receptor positivity in basal cell carcinoma tumor cells (magnification, x100).

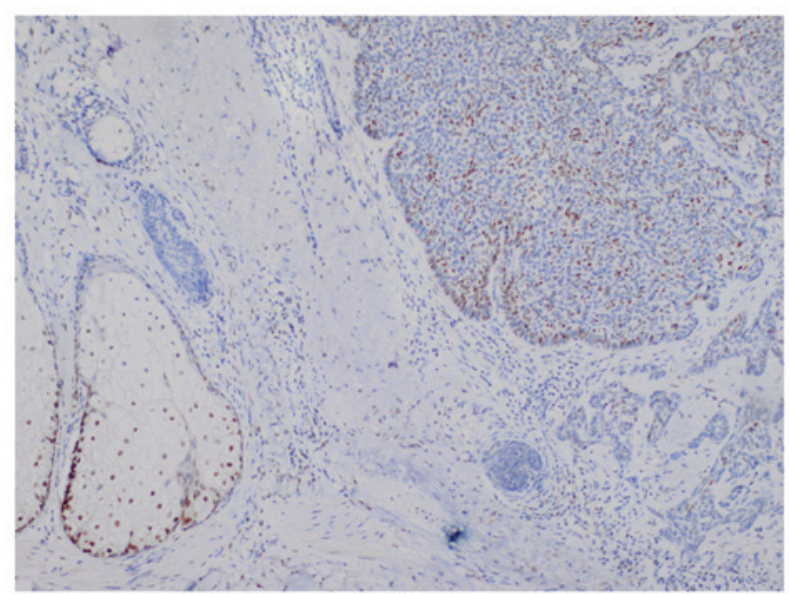

Figure 3. Internal control. Strong nuclear androgen receptor positivity in the basal cell carcinoma on the right. Androgen receptor positivity observed in the sebaceous gland on the left (magnification, x10).

$A R$ expression in BCC and TE patients. None of the TE cases were positive for AR staining, and none of the BCC cases displayed a diffuse nuclear staining pattern (staining, $>50 \%$ ). In total, 16 BCC cases (41\%) were negative for AR staining (AR staining, <1\%), while $23(59 \%)$ BCC cases were positive for AR staining (Table II). Of the AR (+) cases, 5 (21.7\%) were female, and $18(78.3 \%)$ were male (Table III). A total of $17 \mathrm{BCC}$ cases $(43.6 \%)$ displayed ulceration, whereas none of the TE cases displayed ulceration. In addition, $26 \mathrm{BCC}$ (66.7\%) and $5 \mathrm{TE}$ cases (33.3\%) displayed inflammatory infiltrates. Inflammatory infiltrates were more common in BCC cases than in TE cases, however, no statistically significant difference was identified $(\mathrm{P}=0.27)$. Cysts were observed in $21 \mathrm{BCC}(53.8 \%)$ and $3(20 \%)$ TE cases. Cysts were statistically significantly more common in BCC cases than in TE cases $(\mathrm{P}=0.025)$. Clefts were observed in $1 \mathrm{TE}(6.7 \%)$ and $28 \mathrm{BCC}$ cases $(71.8 \%)$ and were thus significantly more common in $\mathrm{BCC}$ cases than in TE cases ( $\mathrm{P}=0.0001$; Table IV). Of the AR (+) BCC cases, $21.7 \%$ displayed stromal CD10 staining, $60.9 \%$ displayed peripheral CD10 tumor cell staining and 30.4\% displayed central CD10 tumor cell staining. By contrast, 17.9\% 
Table IV. Histopathological features of BCC $(n=39)$ and TE $(n=15)$ cases.

\begin{tabular}{lrrr}
\hline $\begin{array}{l}\text { Histopathological } \\
\text { feature }\end{array}$ & BCC, n (\%) & TE, n (\%) & P-value \\
\hline Pigmentation & $15(38.5)$ & $2(13.3)$ & 0.069 \\
Inflammation & $26(66.7)$ & $5(33.3)$ & 0.027 \\
Cyst & $21(53.8)$ & $3(20.0)$ & 0.025 \\
Cleft & $28(71.8)$ & $1(6.7)$ & $<0.001$ \\
Calcification & $5(12.8)$ & $1(6.7)$ & 0.461 \\
\hline
\end{tabular}

BCC, basal cell carcinoma; TE, trichoepithelioma.

Table V. Sensitivity and specificity of CD10 staining, histopathological features and AR (+) in TE and BCC cases.

\begin{tabular}{lrrrrr}
\hline & \multicolumn{2}{c}{ TE } & & \multicolumn{2}{c}{ BCC } \\
\cline { 6 - 7 } \cline { 5 - 6 } Feature & Sens. & Spec. & & Sens. & Spec. \\
\hline CD10 staining & & & & \\
Stromal & 0.400 & 0.282 & & 0.718 & 0.400 \\
Peripheral & 0.733 & 0.590 & 0.410 & 0.733 \\
Central & 0.800 & 0.256 & 0.744 & 0.800 \\
Histopathology & & & & \\
Pigmentation & 0.867 & 0.385 & 0.615 & 0.867 \\
Inflammation & 0.667 & 0.667 & 0.333 & 0.667 \\
Cyst & 0.800 & 0.538 & 0.462 & 0.800 \\
Cleft & 0.933 & 0.718 & 0.282 & 0.933 \\
Calcification & 0.933 & 0.128 & 0.282 & 0.933 \\
AR (+) & $<0.001$ & $<0.001$ & 0.590 & $<0.001$ \\
\hline
\end{tabular}

BCC, basal cell carcinoma; TE, trichoepithelioma; CD10, cluster of differentiation 10; Sens., sensitivity; Spec., specificity; AR (+), androgen receptor positive.

of AR (+) BCC cases were negative for CD10 staining. The results suggest the sensitivities and specificities for the diagnosis of TE, when the differential is BCC. The sensitivity and specificity of CD10 and AR are presented in Table V.

\section{Discussion}

In the present study, AR (+) staining in the BCC group was enriched in the $1-5 \%$ staining group (30.8\% of samples). Furthermore, AR (+) sensitivity was calculated as $59 \%$ and specificity was $0 \%$. The tumor cells in the BCC group displayed a patchy, focal staining pattern. Tumor cells were typically scattered in tumor nodules. A number of tumor islands within the same lesion exhibited no staining, thus, the BCC staining pattern suggested that incisional punch biopsy specimens may exhibit negative staining. Therefore, incisional punch biopsy specimens have the potential to present false-negative staining results, if the total tumor excision is insufficient. In the current study, all cases consisted of excisional biopsy specimens, in order to demonstrate the staining sensitivity and pattern of the tumor cells and stroma in whole lesions. AR staining $<1 \%$ was considered to be negative, thus, $59.9 \%$ of the cases were AR (+). Izikson et al (14) and Katona et al (15) reported AR staining in $78 \%$ and $65 \%$ of their cases, respectively. In addition, Asadi-Amoli et al (16) reported that $33 \%$ of cases were AR (+), whereas Costasche et al (17) reported AR staining in $100 \%$ of cases. In the present study, none of the BCC cases displayed a diffuse nuclear pattern (staining, $>50 \%$ ), a result that was consistent with those of the previous study by Asadi-Amoli et al (16).

Costache et al (17) demonstrated that cytokeratin 20 (CK20) and AR expression aided the differentiation between BCC and TE; however, interpretation was difficult in certain cases. The study also reported that no differences in B cell lymphoma-2 (Bcl-2) and CD34 staining were observed between BCC and TE. By contrast, Kirchmann et al (18) and Illueca et al (19) reported the utility of CD34 as a marker, which is not detected in the stroma of BCC but is present in TE $(18,19)$. A study by Katona et al (15) supported the utility of CK20 and AR expression in the differentiation of BCC and TE. An AR (-), CK20 (+) immunophenotype was sensitive $(87 \%)$ and specific $(100 \%)$ for TE, but was specific (100\%) and moderately sensitive $(61 \%)$ for BCC (15). Furthermore, Choi et al (20) reported that elastic fiber staining and CK15 expression patterns may aid in the differentiation of TE from BCC. Carvalho et al (21) investigated CD23 expression in desmoplastic TE and morpheaform BCC, but observed no statistically significant difference in CD23 expression in these tumors. Furthermore, additional immunohistochemical markers, including Bcl-2, transforming growth factor- $\beta$ and Ber-EP4, may be potentially useful in the differential diagnosis of TE and BCC (22-27).

Sengul et al (28) reported that stromal CD10 immunopositivity in benign cutaneous appendage tumors originating from the hair follicle (trichoepithelioma, trichoblastoma, trichofolliculoma or trichoadenoma) is increased compared with that of $\mathrm{BCC}(\mathrm{P}=0.003)$. However, peripheral, regionally positive $\mathrm{CD} 10$ expression was stronger for $\mathrm{BCC}$ than for benign tumours of cutaneous appendages originating from the hair follicle ( $\mathrm{P}=0.03)$, which is similar to the results of the present study.

Izikson et al (14) observed positive nuclear AR immunostaining in $\sim 78 \%$ of BCCs, whereas positive nuclear AR immunostaining was demonstrated in $\sim 59 \%$ of BCCs. In the present study, $<1 \%$ AR staining was considered to be negative, which may explain the differences in AR percentages reported in these studies. In the same lesions, AR and CD10 staining exhibited distinct staining intensities, and the varied distribution was marked. Incisional punch biopsy specimens may potentially lead to false-negative results. Therefore, AR and CD10 staining are more accurate markers when complete excision of the lesion is performed, particularly in cases where a histopathological differential diagnosis of BCC and TE is difficult.

In the present study, AR positive staining was detected in $59 \%$ of BCC cases, while TE cases exhibited no significant AR staining. In the majority of BCC cases, the expression pattern of AR was focal and nuclear, and the staining typically appeared as scattered clusters and individual tumor cells. None of the TE cases were positive for AR staining. In total, 23 (59\%) of the BCC cases exhibited positive AR staining, and 
of these, 21.7\% exhibited stromal CD10 staining, 60.9\% exhibited peripheral CD10 tumor cell staining and 30.4\% exhibited central tumor cell staining. Furthermore, $17.9 \%$ of the AR (+) $\mathrm{BCC}$ cases were negative for CD10 staining. Additionally, the current study demonstrated that the BCC cases had increased inflammatory infiltrates, cysts, ulceration and clefts compared with those of TE cases. AR positivity of BCC cases was calculated as $59 \%$ sensitive and $0 \%$ specific.

$\mathrm{AR}$ and $\mathrm{CD} 10$ expression demonstrated differing staining intensities and distributions within specific lesions. This observation is significant since incisional punch biopsy specimens may therefore detect negative CD10 and AR staining. On the basis of these results, CD10 and AR staining in incisional punch biopsy specimens may not correctly reflect the diagnosis, which may be accurately determined using a total tumor biopsy. For example, it is possible that specimens obtained by incisional punch biopsy exhibiting negative AR staining may demonstrate AR positivity when evaluated following total excision biopsy.

In conclusion, a differential diagnosis of BCC and TE using the $\mathrm{CD} 10$ and $\mathrm{AR}$ markers requires the use of total excision tumor specimens.

\section{References}

1. Patterson JW: Chapter 31 - Tumors of the epidermis. In: Weedon's Skin Pathology. 4th edition. Elsevier, pp783-835, 2015.

2. Youssef KK, Van Keymeulen A, Lapouge G, Beck B, Michaux C, Achouri Y, Sotiropoulou PA and Blanpain C: Identification of the cell lineage at the origin of basal cell carcinoma. Nat Cell Biol 12: 299-305, 2010.

3. Patterson JW: Chapter 33 - Tumors of cutaneous appendages. In: Weedon's Skin Pathology. 4th edition. Elsevier, pp903-965, 2015.

4. Sawaya ME: Purification of androgen receptors in human sebocytes and hair. J Invest Dermatol 98 (Suppl): S92-S96, 1992.

5. Arits AH, Parren LJ, van Marion AM, Sommer A, Frank J and Kelleners-Smeets NW: Basal cell carcinoma and trichoepithelioma: A possible matter of confusion. Int J Dermatol 47 (Suppl 1): S13-S17, 2008.

6. Bayer-Garner IB, Givens V, Smoller B: Immunohistochemical staining for androgen receptors: A sensitive marker of sebaceous differentiation. Am J Dermatopathol 21: 426-431, 1999.

7. Choudhry R,Hodgins MB, Van der Kwast TH, Brinkmann AO and Boersma WJ: Localization of androgen receptors in human skin by immunohistochemistry: Implications for the hormonal regulation of hair growth, sebaceous glands and sweat glands. J Endocrinol 133: 467-475, 1992.

8. Shikata N, Kurokawa I, Andachi $\mathrm{H}$ and Tsubura A: Expression of androgen receptors in skin appendage tumors: An immunohistochemical study. J Cutan Pathol 22: 149-153, 1995.

9. Dong HY, Gorczyca W, Liu Z, Tsang P, Wu CD, Cohen P and Weisberger J: B-cell lymphomas with coexpression of CD5 and CD10. Am J Clin Pathol 119: 218-230, 2003.

10. Wagoner J, Keehn C and Morgan MB: CD-10 immunostaining differentiates superficial basal cell carcinoma from cutaneous squamous cell carcinoma. Am J Dermatopathol 29: $555-558,2007$.
11. Takahara M, Chen S, Kido M, Takeuchi S, Uchi H, Tu Y, Moroi Y and Furue M: Stromal CD10 expression, as well as increased dermal macrophages and decreased Langerhans cells, are associated with malignant transformation of keratinocytes. J Cutan Pathol 36: 668-674, 2009.

12. Yada K, Kashima K, Daa T, Kitano S, Fujiwara S and Yokoyama S: Expression of CD10 in basal cell carcinoma. Am J Dermatopathol 26: 463-471, 2004.

13. Pham TT, Selim MA, Burchette JL Jr, Madden J, Turner J and Herman C: CD10 expression in trichoepithelioma and basal cell carcinoma. J Cutan Pathol 33: 123-128, 2006.

14. Izikson L, Bhan A and Zembowicz A: Androgen receptor expression helps to differentiate basal cell carcinoma from benign trichoblastic tumors. Am J Dermatopathol 27: 91-95, 2005.

15. Katona TM, Perkins SM and Billings SD: Does the panel of cytokeratin 20 and androgen receptor antibodies differentiate desmoplastic trichoepithelioma from morpheaform/infiltrative basal cell carcinoma? J Cutan Pathol 35: 174-179, 2008.

16. Asadi-Amoli F, Khoshnevis F, Haeri H, Jahanzad I, Pazira R, Shahsiah R: Comparative examination of androgen receptor reactivity for differential diagnosis of sebaceous carcinoma from squamous cell and basal cell carcinoma. Am J Clin Pathol 134: 22-26, 2010.

17. Costache M, Bresch M and Böer A: Desmoplastic trichoepithelioma versus morphoeic basal cell carcinoma: A critical reappraisal of histomorphological and immunohistochemical criteria for differentiation. Histopathology 52: 865-876, 2008.

18. Kirchmann TT, Prieto VG and Smoller BR: CD34 staining pattern distinguishes basal cell carcinoma from trichoepithelioma. Arch Dermatol 130: 589-592, 1994.

19. Illueca C, Monteagudo C, Revert A and Llombart-Bosch A: Diagnostic value of CD34 immunostaining in desmoplastic trichilemmoma. J Cutan Pathol 25: 435-439, 1998.

20. Choi CW, Park HS, Kim YK, Lee SH and Cho KH: Elastic fiber staining and cytokeratin 15 expression pattern in trichoepithelioma and basal cell carcinoma. J Dermatol 35: 499-502, 2008.

21. Carvalho J, Fullen D, Lowe L, Su L and Ma L: The expression of CD23 in cutaneous non-lymphoid neoplasms. J Cutan Pathol 34: 693-698, 2007.

22. Verhaegh ME, Arends JW, Majoie IM, Hoekzema R and Neumann HA: Transforming growth factor-beta and bcl-2 distribution patterns distinguish trichoepithelioma from basal cell carcinoma. Dermatol Surg 23: 695-700, 1997.

23. Smoller BR, Van de Rijn M, Lebrun D and Warnke RA: Bcl-2 expression reliably distinguishes trichoepitheliomas from basal cell carcinomas. Br J Dermatol 131: 28-31, 1994.

24. Basarab T, Orchard G and Russell-Jones R: The use of immunostaining for bcl-2 and CD34 and the lectin peanut agglutinin in differentiating between basal cell carcinomas and trichoepitheliomas. Am J Dermatopathol 20: 448-452, 1998.

25. Poniecka AW and Alexis JB: An immunohistochemical study of basal cell carcinoma and trichoepithelioma. Am J Dermatopathol 21: 332-336, 1999.

26. Abdelsayed RA, Guijarro-Rojas M, Ibrahim NA and Sangueza OP: Immunohistochemical evaluation of basal cell carcinoma and trichepithelioma using Bcl-2, Ki67, PCNA and P53. J Cutan Pathol 27: 169-175, 2000.

27. Swanson PE, Fitzpatrick MM, Ritter JH, Glusac EJ and Wick MR: Immunohistologic differential diagnosis of basal cell carcinoma, squamous cell carcinoma, and trichoepithelioma in small cutaneous biopsy specimens. J Cutan Pathol 25: 153-159, 1998.

28. Sengul D, Sengul I, Astarci MH, Ustun H and Mocan G: CD10 for the distinct differential diagnosis of basal cell carcinoma and benign tumours of cutaneous appendages originating from hair follicle. Pol J Pathol 61: 140-146, 2010. 